International Journal of Advances in Pharmacy and Biotechnology

Vol.2, Issue-2, 2016, 15-31

Research Article

Open Access

I J A P B

ISSN: 2454-8375

\title{
FORMULATION AND EVALUATION OF ORAL FAST DISSOLVING FILMS OF POORLY SOLUBLE DRUG LOPERAMIDE HCI USING TRANSCUTOL HP
}

\author{
Pondugula Sudhakara Reddy*1, GSN Koteswara Rao² and KV Ramana Murthy³ \\ ${ }^{1}$ Hetero Drugs, Hyderabad, Telangana, India. \\ 2Viswanadha Institute of Pharmaceutical Sciences, Visakhapatnam, Andhra Pradesh, India. \\ ${ }^{3}$ A.U. College of Pharmaceutical Sciences, Andhra University, Visakhapatnam, Andhra Pradesh, India. \\ *Corresponding author e-mail: sudhakarpharma@gmail.com
}

Received: 22 September 2016

Revised: 03 October 2016

Accepted: 15 October 2016

\begin{abstract}
:
BCS class II drugs need the enhancement of solubility, hence numerous techniques have been explored in the present literature for the improvement of the solubility. In the present investigation it was tried to study the effect of transcutol HP in increasing the solubility by formulating the OFDFs with transcutol HP and different concentrations of HPMC E series grades indicated that all the prepared formulations are having the physical properties like tensile strength, surface $\mathrm{pH}$, folding endurance, drug release, disintegration time and drug content. The drug release studies also indicated the success of improvement in solubility and dissolution for the films that are prepared in presence of transcutol HP. Based on the results of all the formulations, optimized formulations have confirm the improvement in the solubility, blank formulations were prepared by using similar formula without transcutol HP in the formulation. The results clearly indicated that influence of transcutol HP in optimized formulations shown almost complete drug release within 10 min of time, whereas approximately $50 \%$ of drug release observed after $90 \mathrm{~min}$ of time, hence the effect of transcutol HP was clearly established. The prepared optimized formulations were subjected to stability studies both conditions at accelerated and real time, and results were in concurrence in the stability studies, hence it can be concluded that the formulations are stable.
\end{abstract}

Key words: Transcutol HP, Loperamide HCl, HPMC E5, HPMC E6, HPMC E15, Pectin

\section{INTRODUCTION:}

The objective of the present formulation research is to deliver the drugs at a faster rate and to provide immediate onset of action in a shorter period of time with improved bioavailability. However, poorly soluble drugs pose problems for achieving this goal. The selection of formulation is considered to play an essential role in the development of a successful product of a poorly soluble molecule. The bioavailability can be acknowledged by the formulation at an early stage, then it is necessary to have strategy for maximising the absorption of molecule. Numerous approaches are being followed by the research scientists all over the world to improve the solubility of poorly water soluble molecules with different formulation techniques $^{1-3}$ like complexation, surfactant co-solvent systems, liquisolid systems, lipid systems etc. Rapid mouth disintegrating drug delivery systems were first developed in the late 1970's as substitutes to unit dosage forms like tablets, capsules and syrups or suspensions for paediatric and geriatric patients who are having difficulty in swallowing traditional oral solid dosage forms. In continuation, different range of oral disintegrating tablets were developed and commercialized, which disintegrate in around 60 seconds time after administering in the mouth without water ${ }^{4}$. Wide range of drugs 
like neuroleptics, cardiovascular drugs, analgesics, anti-histamines, anti-asthmatics, anti-diarrheal and erectile dysfunctions can be considered as candidates for oral fast disintegrating dosage forms ${ }^{5}$.

The aim is to increase the solubility and thereby bioavailability of poorly water soluble drugs. For poorly water soluble drugs the rate limiting step for absorption is the disintegration followed by dissolution of the drug, hence the objective of majority of formulation scientists is to improve the disintegration of solid dosage forms followed by dissolution of solid dosage form with different approaches of solid dispersions and different methods of solid dispersions like hot melt extrusion, compression moulding, fusion method and solvent evaporation methods. Oral fast dissolving films contains water soluble polymer which allows the films to quickly hydrate by saliva, adhere to mucosa and disintegrate in seconds followed by releasing of active ingredient for oro-mucosal absorption when administered on the tongue ${ }^{6}$. These films or strips can reportedly incorporate soluble, insoluble or taste masked drug substances. Films are manufactured in large sheet and then divided into individual dosage units by cutting for suitable packaging in a range of pharmaceutically acceptable formats. OFDFs are useful in patients such as bedridden, geriatric, paediatric, developmentally disabled, feeling difficulty in swallowing conventional dosage forms ${ }^{7}$. OFDFs give quick absorption and immediate bioavailability of drugs due to high blood flow thereby permeability of oral mucosa is 4 to 1000 times greater than that of skin absorption ${ }^{8}$.

The general composition of oral films is summarized in Table 1.
Table 1: Composition of oral thin film

\begin{tabular}{lc}
\hline \multicolumn{1}{c}{ Ingredients } & \% used \\
\hline API & Up to 30 \\
Water soluble/swellable & $30-75$ \\
polymer & Up to 6 \\
Saliva stimulating agent & $3-6$ \\
Sweetener & $10-25$ \\
Plasticizer & Quantity sufficient \\
Colour \& Flavour &
\end{tabular}

There are four methods for manufacturing of OFDFs available, those are solvent casting, hot melt extrusion, semisolid casting, solid dispersions. However the most common method used in industry are solvent-casting and hot-melt extrusion methods.

Solvent casting method ${ }^{9,10:}$

OFDFs are preferably formulated using the solvent casting method. In this process initially all the water soluble (plasticizers, sweeteners etc.) ingredients are dissolved in solvent to form a clear solution. Then the active and remaining ingredients are dissolved in smaller amounts in the above aqueous clear solution and then filtered. The entrapped air is removed by vacuum and casted as film on appropriate plates or conveyers which are non-stick and allowed to dry, which is then cut in to pieces with desired size (around $2 \mathrm{~mm}$ ). Advantages of this method are uniformity in thickness, Glossy appearance of film, more flexible and good physical properties of film appearance. The disadvantages with this process are polymers must be soluble in volatile solvent or purified water and stable solution with a reasonable minimum solid.

Hot melt extrusion ${ }^{11}$ :

Hot melt extrusion is a technique where drug, polymer and excipients are mixed together and is extruded under high temperature to form a homogenous liquid mass which is cast on the drying tunnel and the last step is 
slitting of mass to form smooth film. The films are punched, pouched and sealed. The major advantages of this technique are solvent free process, few unit operations involved, not required compressibility properties for API selection, uniformity in dosage forms however, the processing of thermo-labile substances may degrade and leads to poor quality of output with exposure to high temperature during the process, polymer flow property is essential, few polymers available.

\section{MATERIALS AND METHODS}

\section{Materials}

Loperamide $\mathrm{HCl}$, a gift sample from Shreeji Pharma international, Vadodara. Transcutol HP, a gift sample from Gattefosse India PVT. Ltd., Goa. And other ingredients like HPMC E5, HPMC E6, HPMC E15 and pectin, sodium saccharin, orange oil, PEG-400 and citric acid.

\section{Methods}

\subsection{Preparation of oral fast dissolving films (OFDFs):}

The OFDFs were prepared by using solvent casting method in petri plate. In the present investigation it was proposed to prepare film containing $2 \mathrm{mg}$ of drug loperamide $\mathrm{HCl}$ in 1 $\mathrm{cm}^{2}(1 \times 1 \mathrm{~cm})$ film. The amount of drug to be incorporated into the film was calculated based on this assumption and the calculations.

\subsection{Procedure for preparation of loperamide HCl OFDFs:}

Formulae of loperamide $\mathrm{HCl}$ OFDFs are presented in the Table 2. Solvent casting method was used for preparation of films using polymers (HPMC E5, E6, E15 and pectin). Initially the polymer weighed accurately and it was dissolved in half quantity of water and mixed on magnetic stirrer. Loperamide $\mathrm{HCl}$ weighed and dissolved in transcutol HP, citric acid and sodium saccharine both dissolved in remaining amount of water. This solution was added to the polymeric solution and stirred well using a magnetic stirrer to obtain a homogenous solution, followed by the addition of PEG-400 as plasticizer and orange oil. This solution was allowed to stand for 30 min for deaeration of the solution. Solution was then casted in to petri dish and kept in hot air oven for 8-10 hrs at 50ㄷ․ After drying, films were removed with the help of sharp edges. Thus the obtained large film was cut into pieces with the area of $1.0 \mathrm{~cm}^{2}(1 \mathrm{~cm}$ length $\mathrm{x} 1 \mathrm{~cm}$ breadth).

\subsection{EVALUATION OF OFDFs:}

The oral fast dissolving films were evaluated for their dissolution, organoleptic characteristics and mechanical properties like thickness, dryness, tensile strength, folding endurance, transparency, disintegration time, surface $\mathrm{pH}$, moisture loss, moisture uptake and uniformity of drug content.

\subsubsection{Dissolution:}

The dissolution studies were carried out using USP dissolution apparatus type $\mathrm{V}$ (Paddle over disc, Electro lab, Mumbai, India) at $37 \pm 0.5^{\circ} \mathrm{C}$ and $50 \mathrm{rpm}$ using $900 \mathrm{ml} \mathrm{pH} 6.8$ phosphate buffer solution served as medium for loperamide $\mathrm{HCl}$ OFDFs. Each film having 2 $\mathrm{mg}$ of loperamide $\mathrm{HCl}$ was placed in dissolution apparatus.

\subsubsection{Organoleptic properties:}

The organoleptic characters like colour, odour and appearance were examined physically and reported accordingly.

\subsubsection{Thickness:}

Precise film thickness measurements were carried out using calibrated digital micrometre NIKON Digi-Micro screw gauge and then mean average $(n=3)$ is calculated 
subsequently. Uniformity of film weight is calculated in triplicate by cutting the film in 1 $\mathrm{cm}$ length and $1 \mathrm{~cm}$ breadth $(1 \times 1 \mathrm{~cm})$ for determining the weight of film. Thickness of the film measured at five points i.e. from the centre to all the four corners and mean thickness is calculated. It is necessary to determine the uniformity in thickness as it is directly related to accuracy of dose in the film.

\subsubsection{Dryness:}

The ability to adhere a piece of paper pressed between two strips was studied in dryness test $^{12}$. There were eight stages involved in film drying process as per the literature, which were viz. dry-to touch, dry-to-recoat, dry hard, set-to-touch, dust-free, dry-through, tack-free and dry print-free were observed. These tests are used to evaluate dryness of films in pharmaceutical industry especially for orally disintegrating films ${ }^{13}$. Tack-free is the tenacity with which, the strip adheres to a piece of paper that has been pressed into contact with the other strip, instruments are also available for conducting this study.

\subsubsection{Tensile strength:}

Mechanical properties of the film formers are important for film casting on release liners, punching and packaging. Tensile strength is defined as maximum stress applied at which, the film breaks. A quality control test was adapted using number 5 test specimen, tensile strength test was performed using stable micro system's film support rig apparatus to assess strength and elasticity of the prepared films. $1 \mathrm{~cm}^{2}$ film taken and kept between two cup holder and tight them with screws and place it on the film support rig instrument. Depending on the cut film used, different results arose based on the tensile strength of the films. Well established parameters are tensile strength $[\sigma \mathrm{M}=$ maximum tension of test specimen during tensile test] as well as modulus of elasticity in tension. It can be calculated from applied load at rupture divided by the strip cross-sectional area given in the equation below:

Tensile strength $=$ (load at failure $/$ strip thickness $\mathrm{x}$ strip width) $\mathrm{x} 100$

\subsubsection{Folding endurance:}

Folding endurance to determine mechanical properties of film and was measured by repeatedly folding of the film at the same place to the extent where film breaks. The number of times the film is folded without breaking is calculated as the folding endurance value ${ }^{14}$.

\subsubsection{Transparency:}

This parameter was checked simply by visual inspection of films.

\subsubsection{Disintegration time:}

A film was placed onto $2 \mathrm{ml}$ distilled water taken in petri dish. Time taken by the film to dissolve completely is considered as the disintegrating time. The disintegration time is the time when the film starts to break or disintegrates completely, normally disintegration time for oral films s within 2 $\min ^{15}$.

\subsubsection{Dispersion time:}

For determination of in vitro dispersion time, one film with the dimensions $1 \times 1 \mathrm{~cm}$ was placed in a beaker containing $10 \mathrm{ml}$ of $\mathrm{pH} 6.8$ phosphate buffer at $37 \pm 0.5^{\circ} \mathrm{C}$ and the time required for complete dispersion was determined 16 .

\subsubsection{Surface $\mathrm{pH}$ :}

The surface $\mathrm{pH}$ of OFDF was determined to investigate the possibility of any side effects in in vivo. As an acidic or alkaline $\mathrm{pH}$ may cause irritation to the oral mucosa, it was ensured to keep the surface $\mathrm{pH}$ as close to $\mathrm{pH}$ 6.8 (oral cavity $\mathrm{pH}$ ). The $\mathrm{pH}$ of an oral film 
was usually determined by putting the film in petri dish and film was made wet with distilled water and noting $\mathrm{pH}$ by touching the film surface with a $\mathrm{pH}$ paper ${ }^{17}$.

\subsubsection{Moisture loss, moisture uptake:}

Moisture loss determined by finding the initial weight of the film, afterward putting film in a desiccator containing calcium carbonate for about $72 \mathrm{hrs}$. After completion films were taken out and weighed it. Percentage moisture loss is calculated by using the following formula as below ${ }^{16}$.

$\%$ Moisture loss = initial weight - final weight

$$
\text { / initial weight } x 100
$$

Moisture uptake of an oral thin film is determined exposed to environment with a relative humidity $75 \%$ at room temperature for 72 hrs. Percentage moisture uptake is calculated as \% weight gain of the films as per below formula ${ }^{18}$.

$$
\begin{gathered}
\% \text { Moisture uptake }=\text { final weight }- \text { initial } \\
\text { weight } / \text { initial weight } \times 100
\end{gathered}
$$

\subsubsection{Uniformity of drug content:}

Content of oral fast dissolving films were determined by standard assay method taken for individual drug in different pharmacopoeia. This test was performed on 10 samples using different analytical techniques as per the test procedures. The acceptance value of the test is less than 15 in accordance with all pharmacopoeia. A film of size $1 \mathrm{~cm}^{2}$ was cut and kept in $100 \mathrm{ml}$ of volumetric flask containing solvent. This was then shaken in a mechanical shaker till it was dissolved to get a homogeneous solution and then filtered. The drug was determined spectroscopically after appropriate dilution and dilutions were measured at $232 \mathrm{~nm}^{19}$ to get absorbance.

\subsection{CHARACTERIZATION ${ }^{20,21:}$}

\subsubsection{Compatibility studies:}

Drug-polymer compatibility studies were performed by Fourier transform infrared spectroscopy (FTIR) using pressed pellet technique. The pellet was prepared by compression of small pinch of the material with potassium bromide (KBR) and analysed at wave number range $4000-500 \mathrm{~cm}^{-1}$.

\subsubsection{Powder X-ray diffractometry:}

The powder X-ray diffractograms of pure drug loperamide $\mathrm{HCl}$, physical mixtures of optimized formulations, placebo films and optimized film formulation loperamide $\mathrm{HCl}$ were recorded on pXRD with copper radiation in the $2 \theta$ range of $5-80^{\circ}$ at $40 \mathrm{~mA}$ and $45 \mathrm{KV}$ to identify the possible interactions.

\subsubsection{Differential scanning calorimetry (DSC):}

Thermal analysis studies were performed to pure API loperamide $\mathrm{HCl}$ and optimized film formulations of loperamide $\mathrm{HCl}$. The samples were sealed in aluminium pan and heated at $10^{\circ} \mathrm{C} / \mathrm{min}$ rate from $20-200^{\circ} \mathrm{C}$ with empty aluminium pan kept as reference sample.

\subsubsection{Surface texture:}

Surface texture for loperamide $\mathrm{HCl}$ was studied by SEM analysis. The surface morphology of pure API loperamide $\mathrm{HCl}$ and optimized films of loperamide $\mathrm{HCl}$ were observed by scanning electron microscopy.

\section{RESULTS AND DISCUSSION}

\subsection{Drug release of OFDFs:}

Loperamide $\mathrm{HCl}$ OFDFs drug release was more than $80 \%$ for all the formulations within $10 \mathrm{~min}$. The drug release data shown in Table 3 and release profiles in Fig. 1. The drug release in the formulations coded L1 L20 was ranging from $83.96 \%$ to $99.3 \%$ 
within $10 \mathrm{~min}$. Thus from the dissolution studies of all the films developed with different grades of HPMC E series and pectin clearly showed that the drug release was high due to enhanced solubility of the loperamide $\mathrm{HCl}$, which indicated that the solubility of loperamide $\mathrm{HCl}$ was increased in oral fast disintegrating films.

\subsection{Evaluation of oral fast dissolving films:}

Oral fast dissolving films were evaluated for their organoleptic characteristics and mechanical properties like thickness, dryness, tensile strength, folding endurance, transparency, surface texture, disintegration time, uniformity of drug content, and surface $\mathrm{pH}$.

\subsubsection{Organoleptic characteristics:}

The organoleptic characteristics such as description, appearance, and odour were observed for films of both the drugs. They were found to be clear and free from foreign materials and air bubbles without odour.

\subsubsection{Thickness:}

The thickness of the loperamide $\mathrm{HCl}$ OFDFs formulations L1 - L20, developed with HPMC E5, E6 and E15 and pectin were found ranging from $0.095 \mathrm{~mm}$ to $0.372 \mathrm{~mm}$. From the obtained thickness data it was observed that the thickness of the film was increased by increasing in the concentration of the film former. Hence, the thickness of the film was directly proportional to its film former concentration.

\subsubsection{Dryness:}

The dryness of loperamide HCl OFDFs of the formulations L1 - L20 having non-sticky to the papers either side of the film.

\subsubsection{Folding endurance:}

Formulations of loperamide $\mathrm{HCl}$ OFDFs folding endurance for L1-L5 was in the range

www.ijapbjournal.com of 28-50, L6-L10 was in the range of 168-197, L11-L15 was in the range of 260-292 and L16-L20 was in the range of 202-231. The observed folding endurance data of the films developed with various viscosities and concentrations of film formers indicated that the increase in viscosities and concentrations of the film lead to increase in the folding endurance of the films.

\subsubsection{Transparency:}

The transparency of loperamide $\mathrm{HCl}$ OFDFs was observed by placing the films before black background and found that all the films were transparent.

\subsubsection{Disintegrating time:}

Disintegration time for loperamide $\mathrm{HCl}$ OFDFs was found in the range of $22 \mathrm{sec}$ to 56 sec in the films developed with HPMC E5 formulations L1 - L5, 25 sec to $59 \mathrm{sec}$ in films developed with different concentrations of HPMC E6 formulations L6 - L10, formulations L11 - L15 disintegration time was 40 sec to 1 min $10 \mathrm{sec}$ developed with HPMC E15. The formulations L16 - L20 prepared with pectin having different concentrations were ranging from $28 \mathrm{sec}$ - $54 \mathrm{sec}$. Therefore, L6 against L16 formulations were compared the disintegration pattern and time between the formulations with HPMC E6 polymer and pectin polymer and the films disintegration pattern shown as below in Fig. 2 for loperamide $\mathrm{HCl}$ OFDFs at initial, around 15 $20 \mathrm{sec}$ and final completion time snaps were represented.

\subsubsection{Surface pH:}

The surface $\mathrm{pH}$ of the film should be similar to that of saliva i.e. 6.8 as it is being kept in the oral cavity for dissolution for avoiding the irritation. The $\mathrm{pH}$ of loperamide $\mathrm{HCl}$ was measured in triplicate for each sample and found in the range from $6.75-6.85$ with an average of around pH 6.80 which, indicated

\section{IJAPB}


that $\mathrm{pH}$ range was well within the targeted $\mathrm{pH}$ and suitable in oral cavity.

\subsubsection{Moisture loss and moisture uptake:}

The formulated OFDFs were evaluated and calculated \% moisture loss. A reduced \% moisture loss was observed with increase in polymer concentration varying from $6.5 \%$ to $4.00 \% \mathrm{w} / \mathrm{w}$ for loperamide $\mathrm{HCl}$ films. The moisture uptake studies indicated an increase in uptake of moisture with increase in concentration of polymer and found to be in the range of $6.5 \%$ to $9.5 \% \mathrm{w} / \mathrm{w}$ which, may be due to increased hydrophilic nature of polymer with increase in viscosity of the polymer.

\subsubsection{Drug Content:}

The drug content of each film formulations were carried out by standard assay method taken for individual drug in different pharmacopoeia. This test was performed on 10 samples using different analytical techniques as per the test procedures. The acceptance value of the test is less than 15 in accordance with all pharmacopoeia. For loperamide $\mathrm{HCl}$ formulations $\mathrm{L} 1$ - L20 developed with HPMC E5, E6, E15 and pectin having different concentrations the drug content was found in the range from 97.5 to $101.9 \%$. Even though all the formulations drug content within the range, $99.6 \%$ of loperamide $\mathrm{HCl}$ in each film was found in formulations developed with polymers pectin and HPMC E6. The loperamide HCl OFDF's evaluation parameters were shown in Table 4.

\subsection{CHARACTERIZATION:}

\subsubsection{Compatibility study by FTIR:}

The FTIR spectrum of the Loperamide $\mathrm{HCl}$ API and optimized loperamide $\mathrm{HCl}$ formulation L6 FTIR spectra are shown in Fig. 3. The sharp peak at $3414.12 \mathrm{~cm}^{-1}$ represents phenol $\mathrm{O}-\mathrm{H}$ stretching peak; the www.ijapbjournal.com sharp peak obtained at wave number range 1500 - $1600 \mathrm{~cm}^{-1}$ corresponds to $\mathrm{C}-\mathrm{O}$ secondary amide group and a sharp peak obtained at the wave number of $709.83 \mathrm{~cm}^{-1}$ belongs to CCL stretching respectively for pure loperamide $\mathrm{HCl}$. All the characteristic bands of pure loperamide $\mathrm{HCl}$ were observed in optimized loperamide $\mathrm{HCl}$ formulation $\mathrm{L6}$ suggesting that there was compatibility between the drug and other ingredients.

\subsubsection{Powder X-ray diffractometry:}

pXRD data of pure loperamide $\mathrm{HCl}$, physical mixture, placebo film and loperamide $\mathrm{HCl}$ optimized formulation L6 are shown in Fig. 4. The sharp peaks were observed in between the diffraction angles of $10.6^{\circ}$ to $48.75^{\circ}$ clearly indicated that the loperamide $\mathrm{HCl}$ was in crystalline form. There was complete reduction of peak intensity that was observed in optimized loperamide $\mathrm{HCl}$ optimized film formulation L6 indicating that the nature of the loperamide $\mathrm{HCl}$ in film was amorphous in form.

\subsubsection{Differential scanning calorimetry (DSC):}

The pure loperamide $\mathrm{HCl}$ API and loperamide $\mathrm{HCl}$ film optimized formulation L6 DSC thermograms are shown in Fig. 5. A sharp peak obtained between 220-230 $\mathrm{C}$ represents the melting point of loperamide $\mathrm{HCl}$ pure API. The formulation contains a broad peak at the same temperature represents the conversion of crystalline to amorphous nature of the drug and it was also observed that the ingredients used to formulate the film does not shown any interaction with loperamide $\mathrm{HCl}$, that indicated all the ingredients were compatible with the loperamide $\mathrm{HCl}$ API.

\subsubsection{Surface texture:}

Surface texture of the loperamide $\mathrm{HCl}$ films were found to be smooth in nature 
irrespective of the films developed. The pure loperamide $\mathrm{HCl}$ API and loperamide $\mathrm{HCl}$ film optimized formulation L6 Scanning electron microscopy images are shown in Plates 1 \& 2 at different scales. From the obtained SEM images of pure API shown in Plate 1 confirms the crystallinity of loperamide $\mathrm{HCl}$ pure API. The formulation L6 contained a smooth surface represents the conversion of crystalline to amorphous nature of the drug shown in Plate 2.

\subsubsection{Preparation of OFDFs without transcutol HP for the selected drugs:}

From the above results it was concluded that the oral fast dissolving film of L6 of loperamide $\mathrm{HCl}$ respectively gave good results with respect to qualitative and drug release characteristics. Hence, to assess the effect of transcutol HP in increasing the solubility of poorly soluble drugs, films of loperamide $\mathrm{HCl}$ was prepared with the same composition without transcutol HP under similar conditions and are coded as BL6. The prepared OFDFs (BL6) was evaluated for their folding endurance, drug content estimation, disintegration time and dissolution. The prepared films were able to give the satisfactory results with respect to drug content as $100.5 \%$ and folding endurance as 164 of BL6 formulation. The disintegration times were $95 \mathrm{sec}$ and drug

release studies were performed and the results are shown in Table 4 and Fig. 6 in comparison with the films containing transcutol HP.

\section{CONCLUSION}

In the present study the solubility of loperamide $\mathrm{HCl}$ was studied in different solubilizing agents and it was observed that, transcutol HP increased the solubility of loperamide $\mathrm{HCl}$ by 3 to 4 times. Based on the enhancement of solubility when compared with water solubility, it was proposed to prepare the oral fast dissolving films using transcutol HP as solubilizing agent. The films were prepared by using HPMC E series with different viscosity grades and pectin using different drug-polymer concentrations. The prepared films were found to be good quality in nature with respect to the dissolution, thickness, disintegrating time, folding endurance, drug content etc. Based on the characteristics of the film formulations L6 of loperamide $\mathrm{HCl}$ prepared with HPMC E6 gave good results with complete release of drug within 10 mins. Thus it can be concluded that transcutol HP can be used as solubilizing agent for poorly soluble drug like loperamide $\mathrm{HCl}$. The study clearly indicated the influence of transcutol HP in enhancing the solubility of the poorly soluble drugs as the films made without transcutol HP failed to give the desired release characteristics within $10 \mathrm{~min}$. 
Table 2: Formulae of loperamide HCl OFDFs

\begin{tabular}{ccccccccccccccccccccc}
\hline Ingredients (mg) & L1 & L2 & L3 & L4 & L5 & L6 & L7 & L8 & L9 & L10 & L11 & L12 & L13 & L14 & L15 & L16 & L17 & L18 & L19 & L20 \\
\hline Loperamide HCl & 36 & 36 & 36 & 36 & 36 & 36 & 36 & 36 & 36 & 36 & 36 & 36 & 36 & 36 & 36 & 36 & 36 & 36 & 36 & 36 \\
HPMC E5 & 330 & 355 & 380 & 405 & 430 & - & - & - & - & - & - & - & - & - & - & - & - & - & - & - \\
HPMC E6 & - & - & - & - & - & 330 & 355 & 380 & 405 & 430 & - & - & - & - & - & - & - & - & - & - \\
HPMC E15 & - & - & - & - & - & - & - & - & - & - & 330 & 355 & 380 & 405 & 430 & - & - & - & - & - \\
$\quad$ Pectin & - & - & - & - & - & - & - & - & - & - & - & - & - & - & - & 330 & 355 & 380 & 405 & 430 \\
Citric acid & 66 & 66 & 66 & 66 & 66 & 66 & 66 & 66 & 66 & 66 & 66 & 66 & 66 & 66 & 66 & 66 & 66 & 66 & 66 & 66 \\
Sodium saccharine & 55 & 55 & 55 & 55 & 55 & 55 & 55 & 55 & 55 & 55 & 55 & 55 & 55 & 55 & 55 & 55 & 55 & 55 & 55 & 55 \\
Transcutol HP & 1 & 1 & 1 & 1 & 1 & 1 & 1 & 1 & 1 & 1 & 1 & 1 & 1 & 1 & 1 & 1 & 1 & 1 & 1 & 1 \\
PEG-400 & 1 & 1 & 1 & 1 & 1 & 1 & 1 & 1 & 1 & 1 & 1 & 1 & 1 & 1 & 1 & 1 & 1 & 1 & 1 & 1 \\
Orange oil & 0.25 & 0.25 & 0.25 & 0.25 & 0.25 & 0.25 & 0.25 & 0.25 & 0.25 & 0.25 & 0.25 & 0.25 & 0.25 & 0.25 & 0.25 & 0.25 & 0.25 & 0.25 & 0.25 & 0.25 \\
P. water (ml) & 13 & 13 & 13 & 13 & 13 & 13 & 13 & 13 & 13 & 13 & 13 & 13 & 13 & 13 & 13 & 13 & 13 & 13 & 13 & 13
\end{tabular}
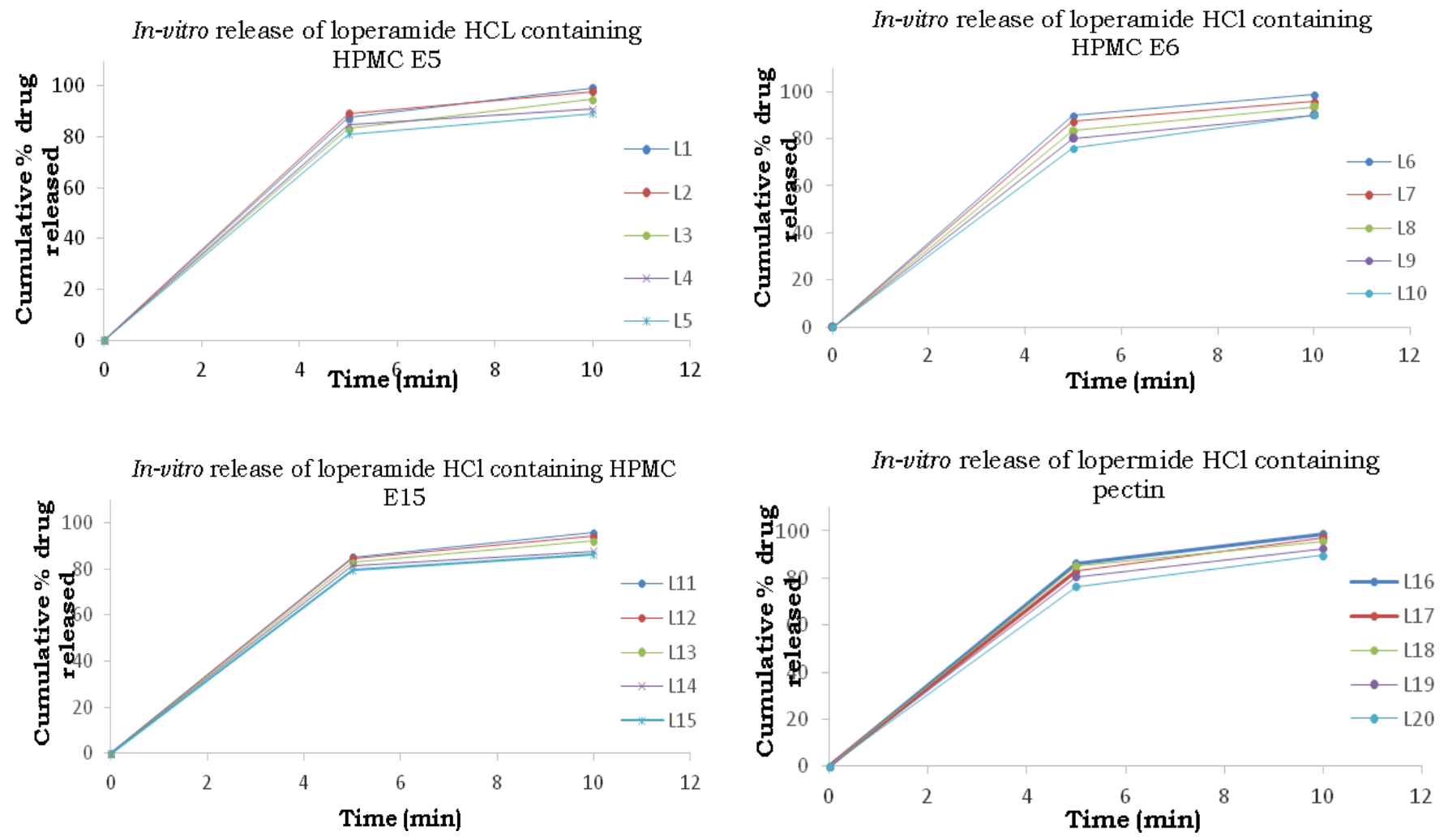

Fig. 1: Dissolution profiles of loperamide HCl OFDFs 
Table 3: Evaluation parameters of loperamide HCl OFDFs

\begin{tabular}{|c|c|c|c|c|c|c|c|}
\hline Formulation & $\begin{array}{l}\text { Thickness } \\
\text { (mm) }\end{array}$ & $\begin{array}{c}\text { Folding } \\
\text { endurance }\end{array}$ & Transparency & $\begin{array}{l}\text { Surface } \\
\text { texture }\end{array}$ & $\begin{array}{c}\text { Disintegration } \\
\text { time }\end{array}$ & $\begin{array}{c}\text { Surface } \\
\text { pH }\end{array}$ & $\begin{array}{c}\text { Drug } \\
\text { content } \\
(\%)\end{array}$ \\
\hline L1 & 0.095 & 28 & Transparent & Smooth & $22 \mathrm{sec}$ & 6.80 & 99.0 \\
\hline $\mathrm{L} 2$ & 0.112 & 30 & Transparent & Smooth & $32 \mathrm{sec}$ & 6.85 & 98.6 \\
\hline L3 & 0.152 & 45 & Transparent & Smooth & $38 \mathrm{sec}$ & 6.80 & 100.0 \\
\hline L4 & 0.184 & 49 & Transparent & Smooth & $44 \mathrm{sec}$ & 6.80 & 97.5 \\
\hline L5 & 0.187 & 50 & Transparent & Smooth & $56 \mathrm{sec}$ & 6.75 & 99.0 \\
\hline L6 & 0.176 & 168 & Transparent & Smooth & $25 \mathrm{sec}$ & 6.80 & 99.6 \\
\hline L7 & 0.193 & 171 & Transparent & Smooth & $37 \mathrm{sec}$ & 6.80 & 99.4 \\
\hline L8 & 0.222 & 184 & Transparent & Smooth & $42 \mathrm{sec}$ & 6.75 & 101.5 \\
\hline L9 & 0.248 & 192 & Transparent & Smooth & $48 \mathrm{sec}$ & 6.85 & 98.6 \\
\hline L10 & 0.278 & 197 & Transparent & Smooth & $59 \mathrm{sec}$ & 6.80 & 101.9 \\
\hline L11 & 0.278 & 260 & Transparent & Smooth & $40 \mathrm{sec}$ & 6.85 & 99.0 \\
\hline $\mathrm{L} 12$ & 0.284 & 271 & Transparent & Smooth & $47 \mathrm{sec}$ & 6.80 & 100.7 \\
\hline L13 & 0.301 & 278 & Transparent & Smooth & $54 \mathrm{sec}$ & 6.85 & 98.4 \\
\hline L14 & 0.321 & 289 & Transparent & Smooth & $1 \mathrm{~min}$ & 6.75 & 101.6 \\
\hline L15 & 0.392 & 292 & Transparent & Smooth & $1 \mathrm{~min} 10 \mathrm{sec}$ & 6.80 & 99.4 \\
\hline L16 & 0.146 & 202 & Transparent & Smooth & $28 \mathrm{sec}$ & 6.80 & 99.6 \\
\hline L17 & 0.184 & 210 & Transparent & Smooth & $35 \mathrm{sec}$ & 6.80 & 99.2 \\
\hline L18 & 0.214 & 217 & Transparent & Smooth & $43 \mathrm{sec}$ & 6.85 & 100.7 \\
\hline L19 & 0.271 & 226 & Transparent & Smooth & $50 \mathrm{sec}$ & 6.75 & 98.8 \\
\hline L20 & 0.277 & 231 & Transparent & Smooth & $54 \mathrm{sec}$ & 6.75 & 99.8 \\
\hline
\end{tabular}

Table 4: Cumulative \% drug released vs. time from loperamide HCl OFDFs

Formulation code

L1

L2

L3

L4

L5

L6

L7

L8

L9

L10

L11

L12

L13

L14

L15

L16

L17

L18

L19

L20
Time (min)

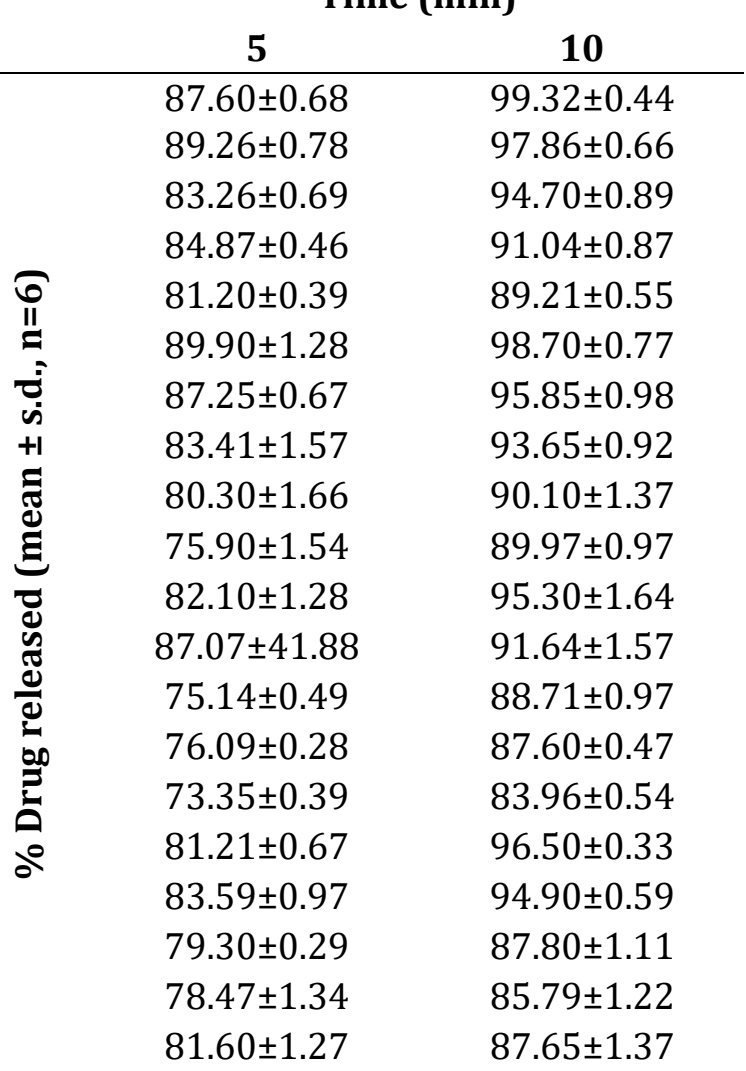


HPMC E6 based formulation

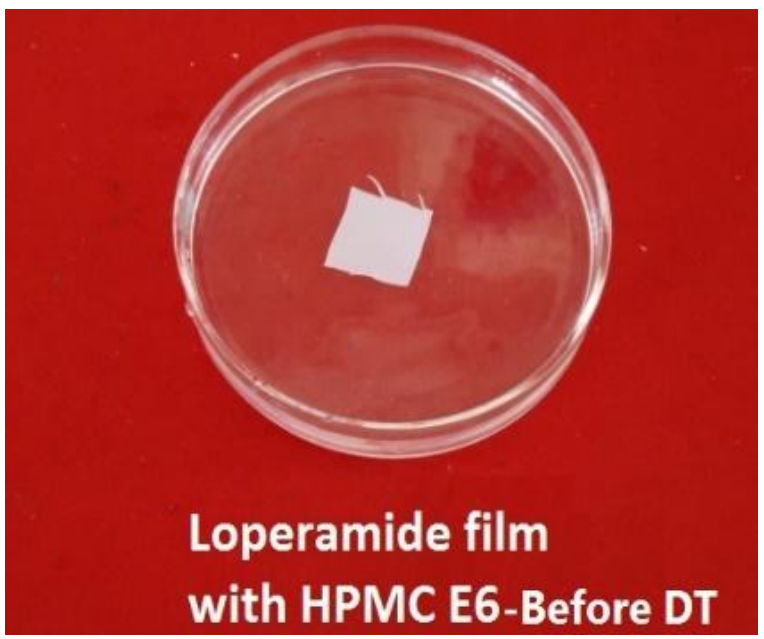

Before disintegration

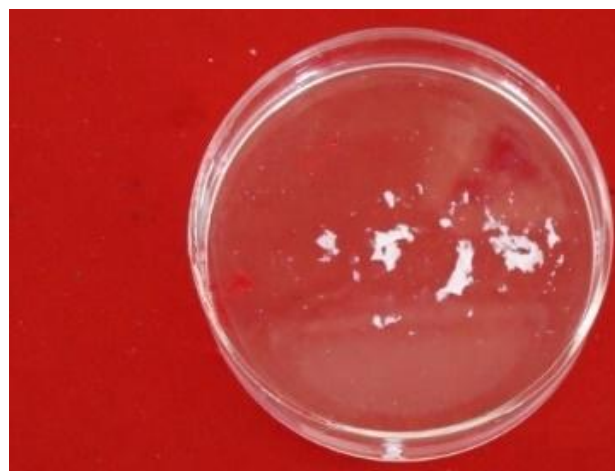

Loperamide Film with HPMC E6 - During DT

During disintegration (15-20 sec)

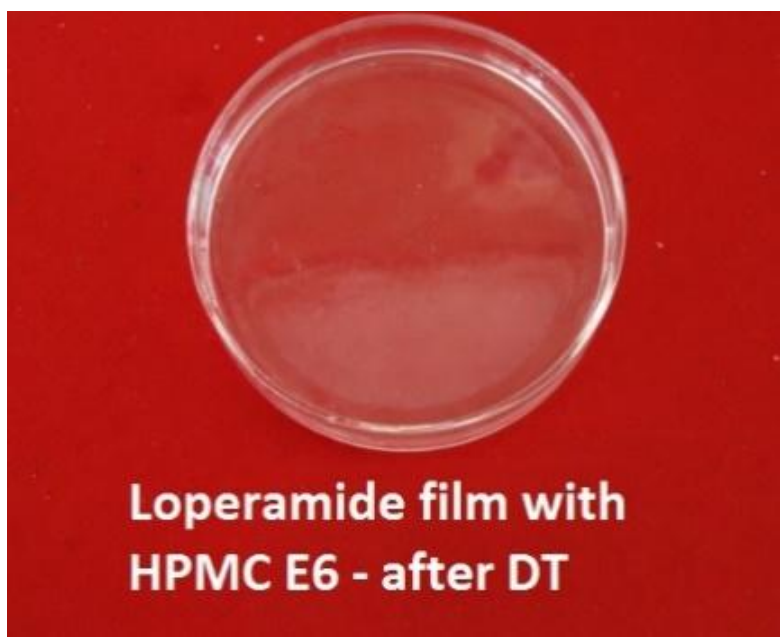

After disitegration
Pectin based formulation

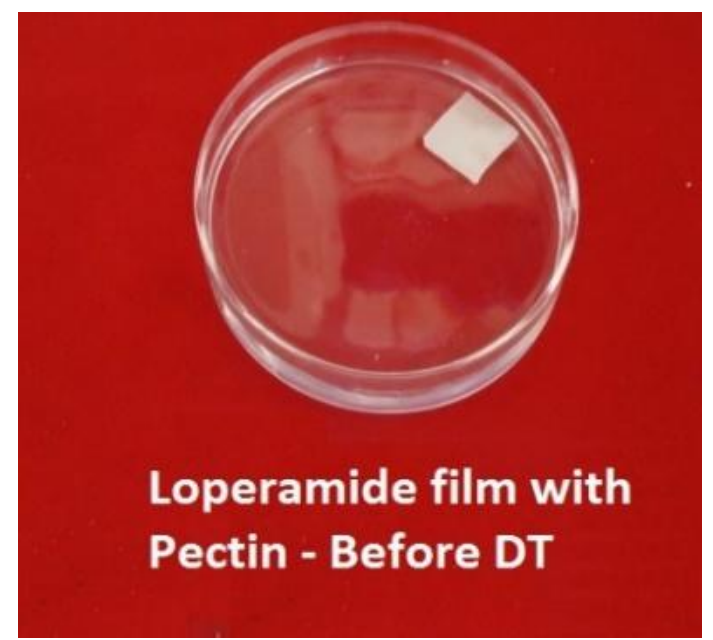

Before disintegration

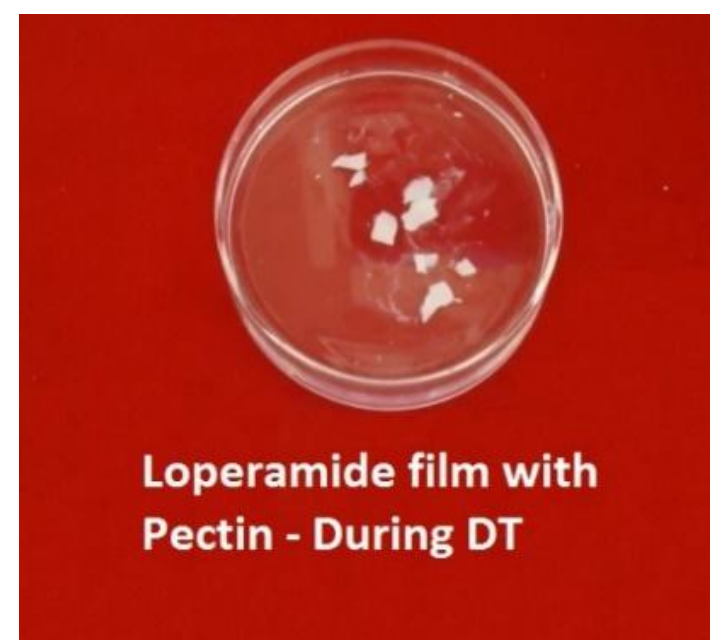

During disintegration (15-20 sec)

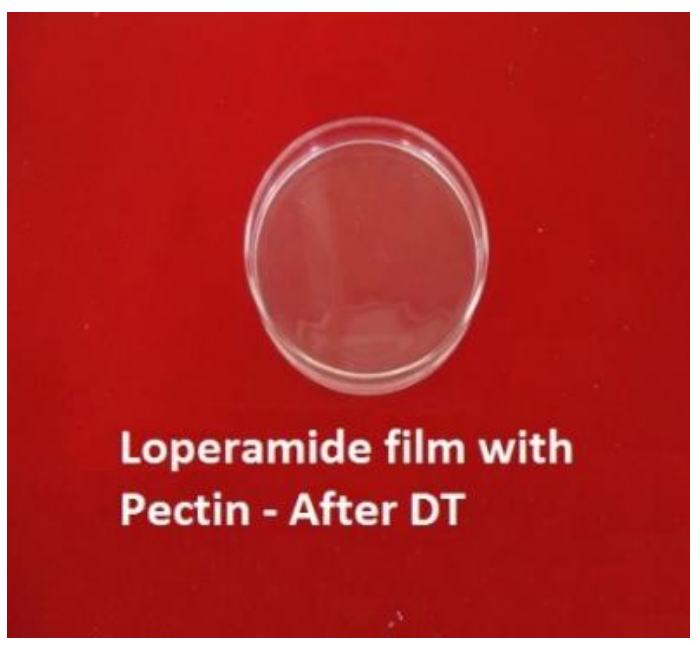

After disintegration

Fig. 2: Loperamide HCl OFDFs disintegrating pattern of L6 \& L16 formulations 

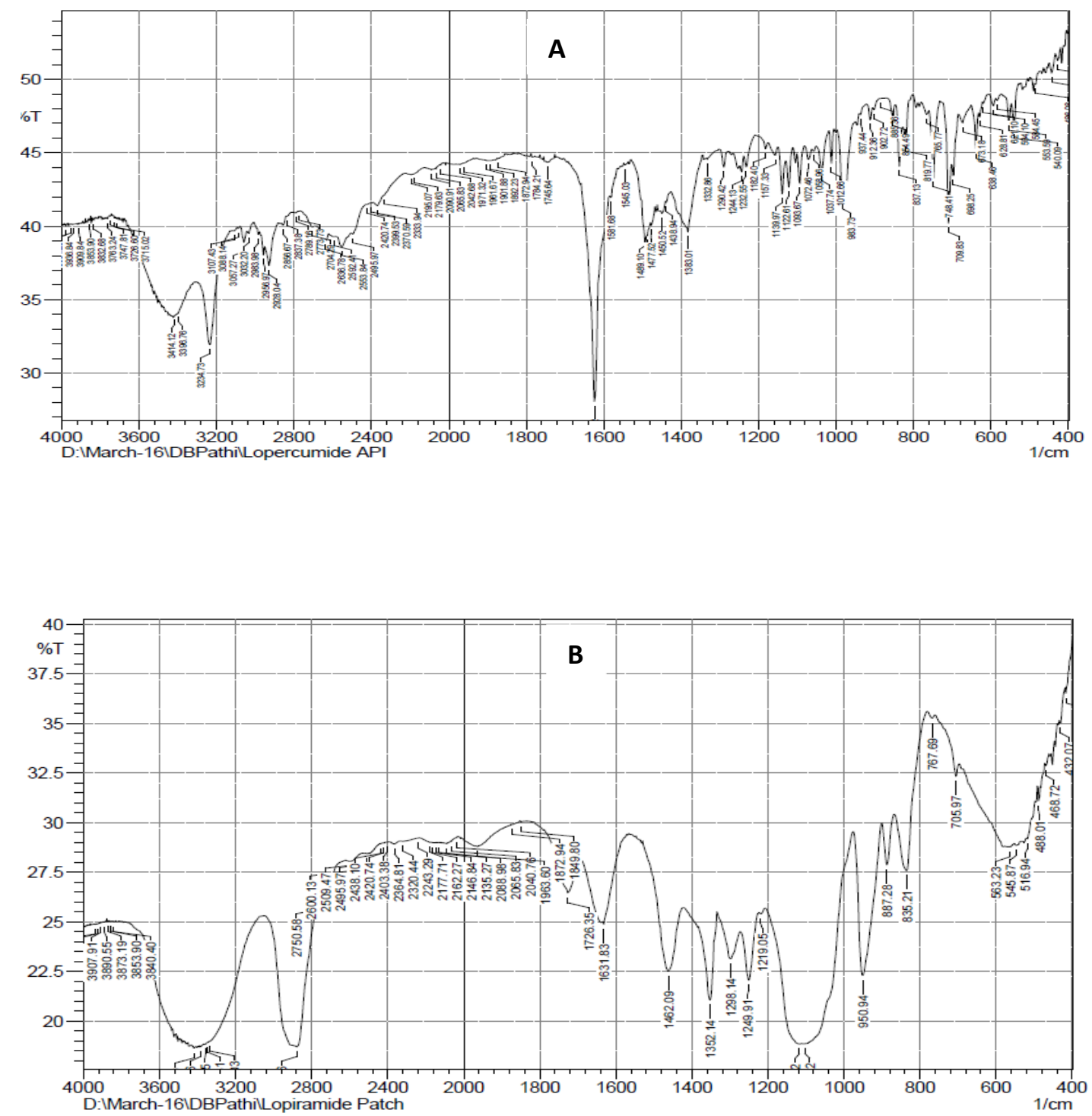

Fig. 3: FTIR spectra of A) loperamide HCl B) loperamide HCl OFDF formulation L6 

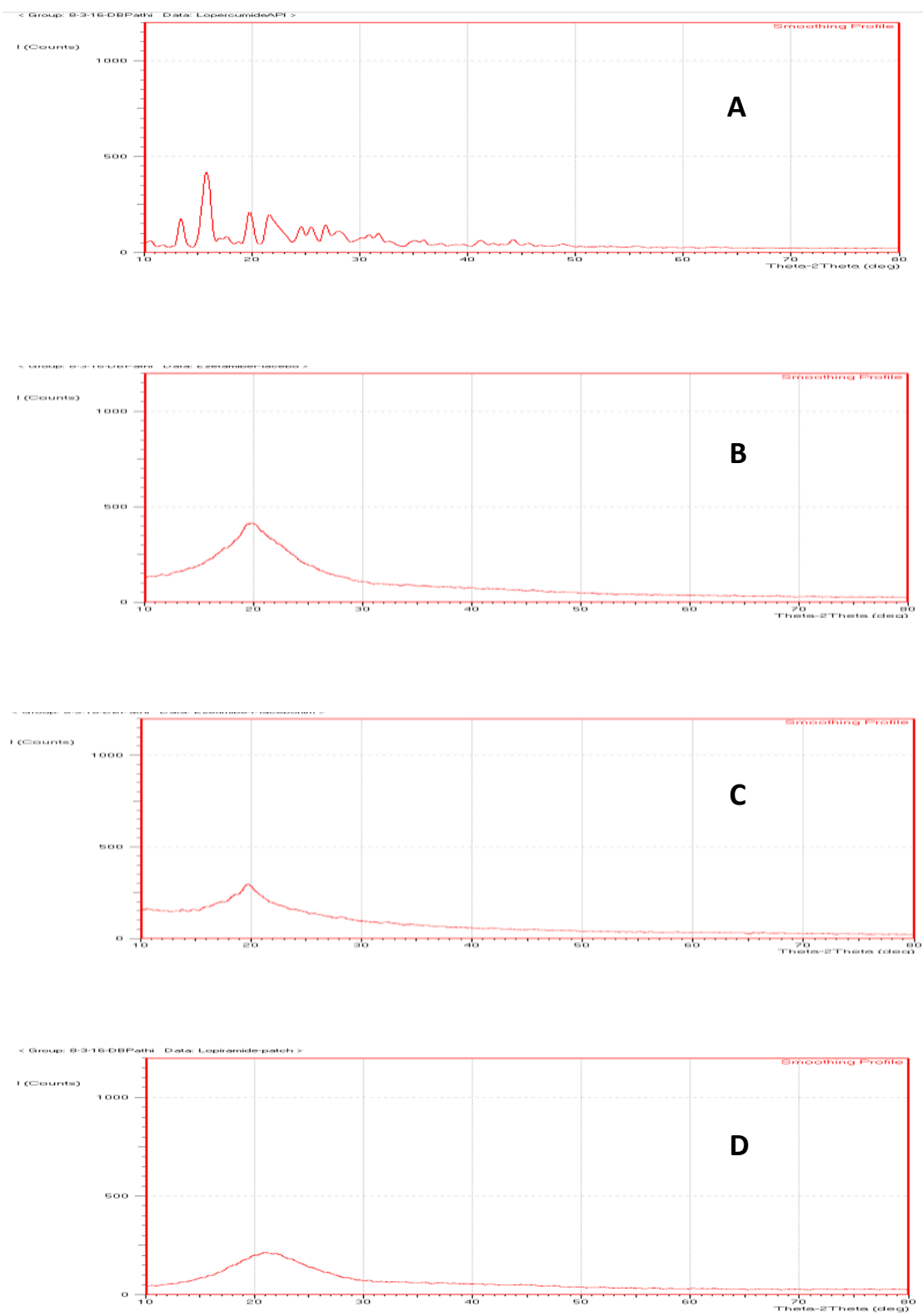

Fig. 4: X-ray diffractograms of A) loperamide $\mathrm{HCl} \mathrm{B}$ ) loperamide $\mathrm{HCl}$ physical mixture C) loperamide $\mathrm{HCl}$ placebo film D) loperamide HCl OFDF formulation L6 

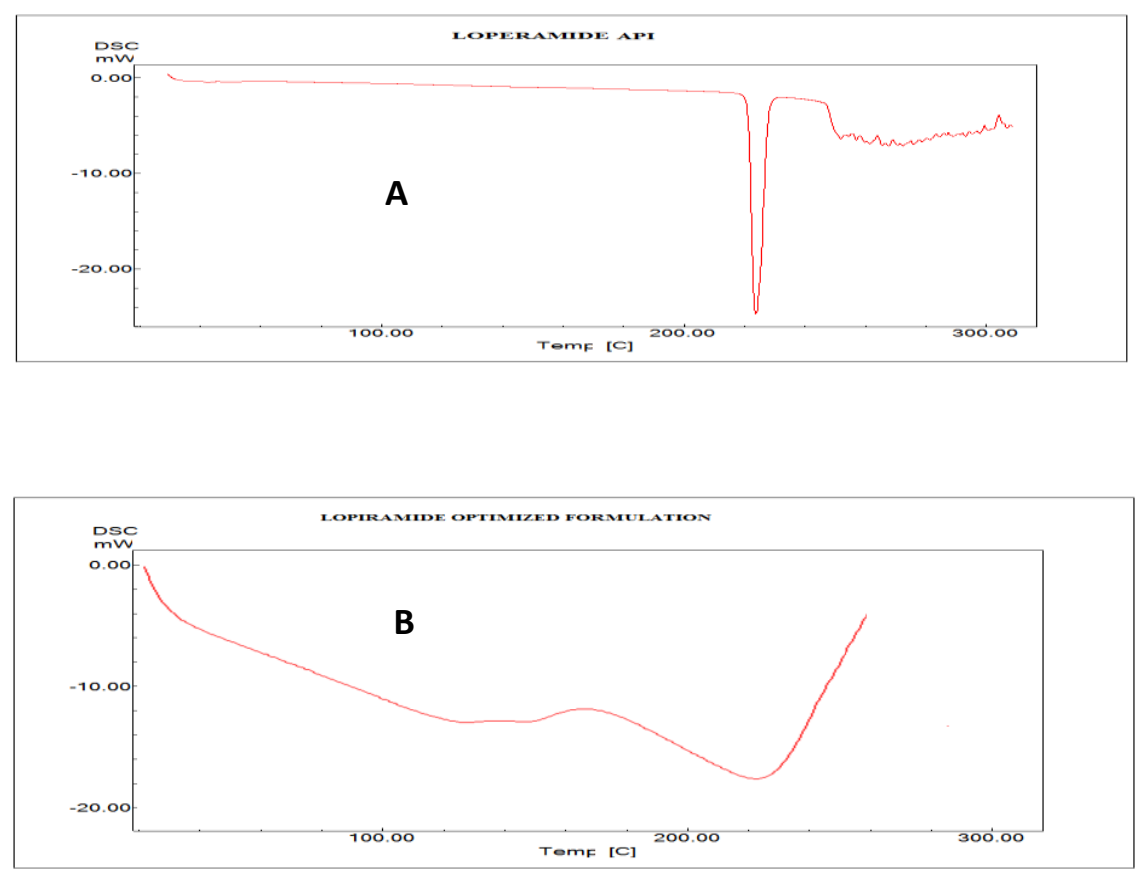

Fig. 5: DSC thermograms of A) loperamide $\mathrm{HCl}$ B) loperamide HCl OFDF formulation L6
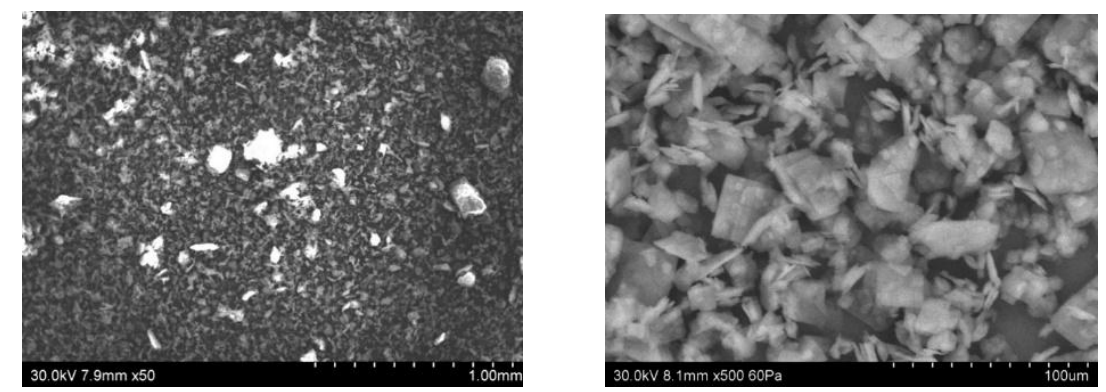

Plate 1: SEM images of loperamide $\mathrm{HCl}$ pure API
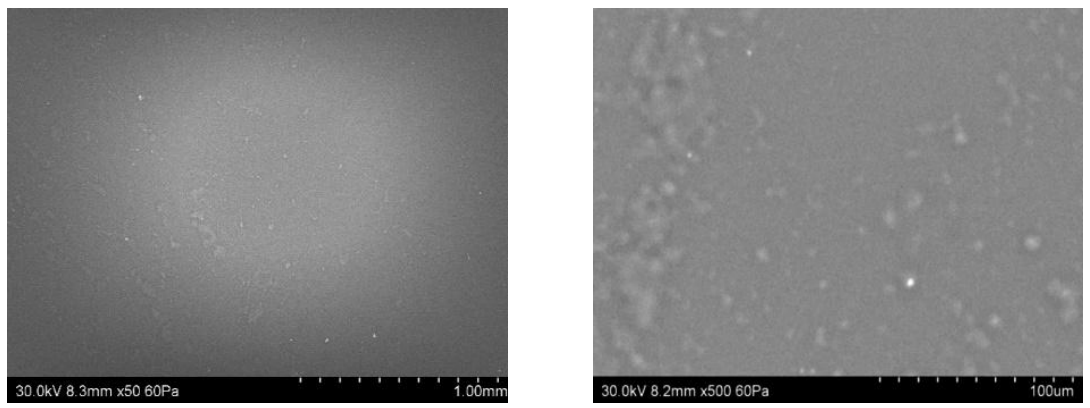

Plate 2: SEM images of loperamide HCl OFDF formulation L6 
Table 5: Comparative dissolution loperamide $\mathrm{HCl}$ optimized films with and without transcutol HP films in pH 6.8

\begin{tabular}{ccc}
\hline \multirow{2}{*}{ Time (min) } & \multicolumn{2}{c}{ \% Drug released (mean \pm s.d., $\mathbf{n = 6 )}$} \\
\cline { 2 - 3 } & L6 & BL6 \\
\hline 5 & $89.9 \pm 1.28$ & $18.2 \pm 5.12$ \\
10 & $98.7 \pm 0.77$ & $24.8 \pm 5.56$ \\
15 & $99.3 \pm 0.82$ & $29.5 \pm 4.35$ \\
30 & $99.1 \pm 0.56$ & $37.4 \pm 1.98$ \\
45 & $98.9 \pm 0.66$ & $39.6 \pm 2.12$ \\
60 & $98.7 \pm 0.68$ & $45.9 \pm 1.12$ \\
90 & $98.7 \pm 0.56$ & $50.1 \pm 0.48$ \\
\hline
\end{tabular}

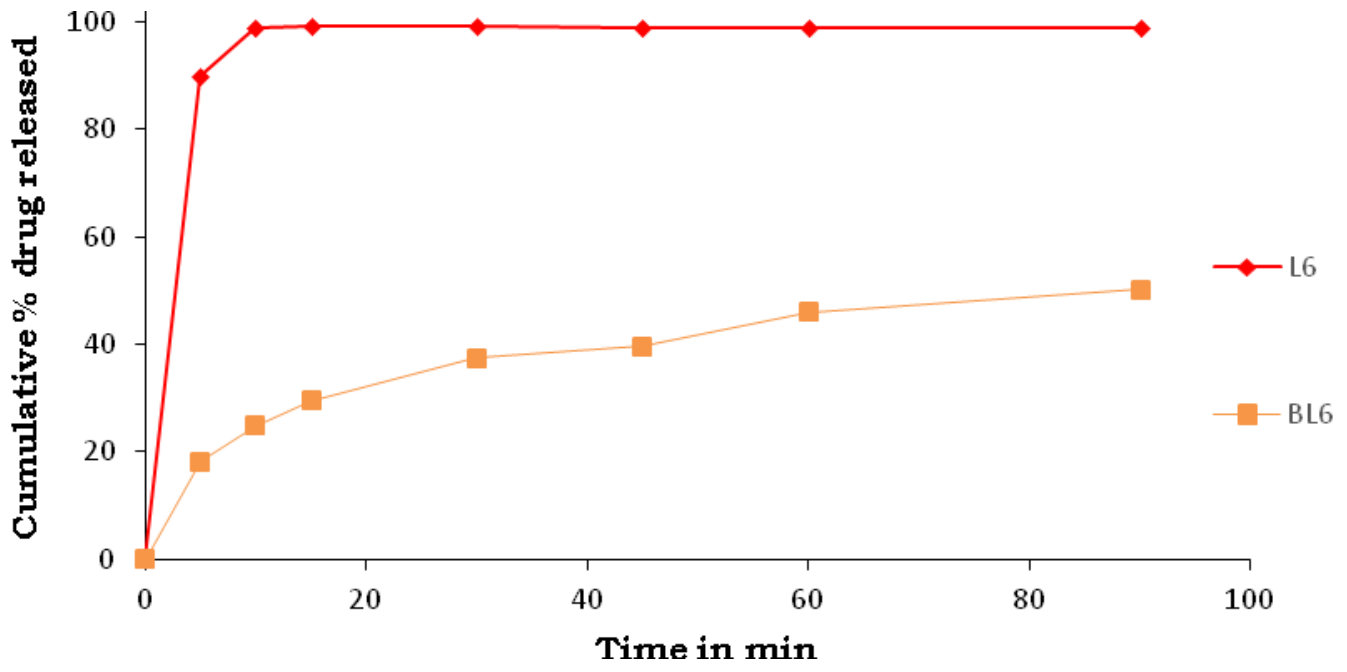

Fig. 6: Comparative dissolution profiles of loperamide $\mathrm{HCl}$ optimized films with and without transcutol HP films

\section{ACKNOWLEDGEMENT}

The authors are thankful to the management and staff of Vaageswari College of Pharmacy for their support in the research carriedout.

\section{REFERENCES}

1.Colin W. Pouton, Formulation of poorly water soluble drugs for oral administration: physicochemical and physiological issues and the lipid formulation classification system, European Journal of Pharmaceutical www.ijapbjournal.com
Sciences., 2006, 29(3-4): 278-287.

2.Michael Hite, Stephen Turner and Cathy Federici, Part 1: Oral delivery of poorly soluble drugs, Pharmaceutical Manufacturing and Packing Sourcer., 2003, Summer 03 issue.

3.Y.S.R. Krishnaiah, Pharmaceutical technologies for enhancing oral bioavailability of poorly soluble drugs, Journal of Bioequvalence and Bioavailability., 2010, 2(2): 28-36.

4.Rajni Bala, Pravin Pawar, Sushil Khanna 
and Sandeep Arora, Orally dissolving strips: A new approach to oral drug delivery system, Int J Pharm Investig., 2013, 3(2): 67-76.

5.R.P. Dixit and S.P. Puthli, Oral strip technology: Overview and future potential, J of Cont Release., 2009, 139(2): 94-107.

6.Aditya Dinge and Mangal Nagarsenker, Formulation and evaluation of fast dissolving films for delivery of triclosan to the oral cavity, AAPS Pharm Sci Tech., 2008, 9(2): 349-356.

7.Alpesh R. Patel, Dharmendra S. Prajapati and Jignyasha A. Raval, Fast dissolving films (FDFS) as a newer venture in fast dissolving dosage forms, International Journal of Drug Development \& Research., 2010, 2(2): 232-246.

8.Hema Chaudhary, Samita Gauri, Permender Rathee and Vikash Kumar, Development and optimization of fast dissolving oro-dispersible films of granisetron $\mathrm{HCl}$ using Box-Behnken statistical design, Bulletin of Faculty of Pharmacy., Cairo University, 2013, 51(2): 193-201.

9.Dhagla R. Choudhary, Vishnu A. Patel, Usmangani K. Chhalotiya, Harsha V. Patel and Alisgar J. Kundawala, Development and characterization of pharmacokinetic parameters of fast-dissolving films containing levocetirizine, Sci Pharm., 2012, 80(3): 779-787.

10.Reeta Rani Thakur and Sonia Narwal, Orally disintegrating preparations: Recent advancement in formulation and technology, J Drug Deliv Therap., 2012, 2(3): 87-96.

11.Franscesco Cilurzo, Paola Minghetti, Andrea Como and Luisa Montanari, "Maltodextrin fast-dissolving film: A feasibility study", http://www.tecnovasrl.it/download/film@EUFEPS051.pdf
12.Hema Chaudhary, Samita Gauri, Permender Rathee and Vikash Kumar, Development and optimization of fast dissolving oro-dispersible films of granisetron $\mathrm{HCl}$ using Box-Behnken statistical design, Bullet. Faculty Pharm., Cairo University, 2013, 51: 193-201.

13.Muhammad Irfan, Sumeira Rabel, Quratulain Bukhtar, Muhammad Imran Qadir, Farhat Jabeen and Ahmed Khan, Orally disintegrating films: A modern expansion in drug delivery system, Saudi Pharmaceutical Journal., 2015, 1-10.

14.Chandra Kala Singh, Vishnu Tiwari, Ravi Shankar, Chandra Prakash Mishra, Sarvesh Jain, Sandeep Jain and Sandhya Jaiswal, A short review on oral fast dissolving film containing cefpodoxime proxetil nanoparticle, World Journal of Pharmacy and Pharmaceutical Sciences., 2015, 5(1): 1549-1577.

15.Pallavi C. Patil, S.K. Shrivastava, S. Vaidehi and P. Ashwini, Oral fast dissolving drug delivery system: A modern approach for patient compliance, Int. J. Drug Regulat Affairs., 2014, 2(2): 49-60.

16.Y.X. Bi, H. Sunada, Y. Yonezawa and K. Danjo, Evaluation of rapidly disintegrating tablets by direct compression method. Drug Develop Ind Pharm, 1999, 25: 571581.

17.S.K. Yellanki, S. Jagtap and R. Masareddy, Disso film: A novel approach for delivery of phenobarbital; design and characterization, J. Young Pharm., 2011, 3(3): 181-188.

18.Ashish Prakash Gorle and Suredra Ganeshlal Gattani, Design and evaluation of polymeric ocular drug delivery system, Chem Pharm Bull (Tokyo), 2009, 57(9): 914-919.

19.Rajni Bala, Pravin Pawar, Sushil Khanna and Sandeep Arora, Orally disintegrating strips: A new approach to oral drug 
delivery system, Int J Pharm Investi., 2013, 3(2): 67-76.

20.KM Maheswari, Pavan Kumar, Devineni, Sravanthi Deekonda, Salma Shaik, Naga Pravallika Uppala and Buchi N. Nalluri, Development and evaluation of mouth dissolving films of amlodipine besylate for enhanced therapeutic efficacy, Journal of pharmaceutics, article ID 520949, 2014, 1-10.

21.Aditya Dinge and Mangal Nagarsenker, Formulation and evaluation of fast dissolving films for delivery of triclosan to the oral cavity, AAPS Pharm Sci Tech., 2008, 9(2): 349-356.

How to cite this article:

Pondugula Sudhakara Reddy et. al., Formulation and evaluation of oral fast dissolving films of poorly soluble drug loperamide HCl using Transcutol HP. Int. J. Adv. Pharm. Biotech., 2016; 2(2): 15-31. 